\title{
A Maximum Likelihood Approach for 2-D to 3-D Registration
}

\author{
Jay B. West, Rasool Khadem, Shao Chin, and Ramin Shahidi
}

Cbyon Inc., 2275 E. Bayshore Road \#101, Palo Alto, CA 94303

\begin{abstract}
In this paper, we examine the problem of point-based registration in which a pair of two-dimensional projective views of an object is used to reconstruct the three-dimensional position of given points within the object. The particular application we consider is that of integrating preoperative, three-dimensional information, for example that provided by a CT scan, with the two-dimensional information given by intraoperative fluoroscopy. To facilitate registration, before the preoperative scan we embed small, removable spheres in the patient's anatomy proximal to the site of proposed surgery; the spheres remain in place until the image-guided phase of the operation is complete.
\end{abstract}

\section{Introduction}

Once the three dimensional position of the fiducial spheres in physical space has been calculated, the image-to-physical registration step is treated as a simple rigid-body, point-based problem. There is a well-known closed-form solution [1] that gives the rotation and translation which minimizes the sum of squared distances between corresponding points in image and physical space.

We present here a refinement to the traditional method of reconstructing the three-dimensional physical positions of the spheres from the two projective views. Using a maximum likelihood approach linked to a statistical error model for localizing the projections of the spheres in the fluoroscope images, we show how to derive the most likely configuration of the spheres in physical space.

\section{Methods}

Fluoroscopic images are formed when X-rays are emitted from a source, pass through an object, and are captured by an image intensifier on the other side of the object. By localizing the projection of one of the fiducial spheres in a fluoroscope image, we infer that the physical location of the sphere is at some point on the line joining the source to the point on the image intensifier corresponding to the sphere's projection. If there were no distortion in the images, and if we could localize the projections of the spheres with perfect accuracy, the physical space localization problem would be reduced to that of taking two fluoroscope images at different orientations and finding the intersections of the spheres' projection lines. 
We use a calibration jig containing spheres in a known configuration in order to correct for the "pincushion" and ambient magnetic field related distortion common in fluoroscope images. However, because of the finite pixel size and digital nature of the images, it is not possible to localize perfectly the projections of the fiducial spheres. We assume that, for each of the spheres in each of the projections, the distances of the localized positions from the true positions are drawn from a Gaussian distribution with zero mean and standard deviation $\sigma$.

Assuming that the X-ray source is at a distance $f$ from the image intensifier, and letting $z$ represent the distance of a fiducial sphere from the source, we have that the localized position of a sphere at this distance from the source is distributed about the true position with a Gaussian distribution having zero mean and standard deviation $\sigma z / f$. If we denote by $L_{1}$ and $L_{2}$ the derived lines between the projections of the spheres in each view and the source, the traditional approach is to write the three dimensional position of the sphere as the center point of the line $P$ perpendicular to, and joining, $L_{1}$ and $L_{2}$. Instead, we choose the point that maximizes the product of the probability density functions. From intuitive geometry, it is clear that the optimal choice of point will lie on $P$. We let $z_{1}$ be the distance from the source of the intersection of $P$ with $L_{1}$, and $z_{2}$ the same for $L_{2}$. We write $D$ as length of the line $P$. Then we wish to choose a point on $P$ at distance $d$ from the intersection of $P$ and $L_{1}$ to minimize

$$
\exp \left(-d^{2} f^{2} / 2 \sigma^{2} z_{1}^{2}\right) \exp \left(-(D-d)^{2} f^{2} / 2 \sigma^{2} z_{2}^{2}\right)
$$

Simple calculus reveals that the optimal choice of $d$ is

$$
d=z_{1}^{2} /\left(z_{1}^{2}+z_{2}^{2}\right)
$$

\section{Conclusion}

We have introduced a refinement to the traditional method of reconstructing three dimensional information from a pair of projective views. We have shown that this method may be used to give the most likely spatial positions of fiducial spheres, thus allowing a potential improvement in the registration of three dimensional preoperative images to the physical space of the operating room when intraoperative fluoroscopy is used. In practice, this refinement may give an improvement of the order of $2 \mathrm{~mm}$ in localization accuracy.

\section{References}

1. P. H. Schönemann, "A generalized solution of the orthogonal Procrustes problem", Psychometrika, vol. 31, pp. 1-10, 1966. 